\title{
Effect of Magnetic Field in Magnetic Separation Column on Particle Separation Process Based on Numerical Simulation
}

\author{
Shun Cao ${ }^{1,2}$, Dezhou Wei ${ }^{1}$ and Jinfeng $\mathrm{Li}^{2, *}$ \\ ${ }^{1}$ School of Resources and Civil Engineering, Northeastern University, Shenyang 110819, China, \\ ${ }^{2}$ College of Information Engineering, Shenyang University of Chemical Technology, Shenyang 110142, China
}

Received 9 February 2019; Accepted 29 April 2019

\begin{abstract}
The magnetic field in the magnetic separation column is a key factor in determining the separation process of mineral particles. The limited data points and low accuracy of measurement of the magnetic field cause difficulty in estimating the relationship between the magnetic field and particle separation process. A calculation model of the magnetic field of the magnetic separation column was proposed in this study to explore its effect on the particle separation process. The calculation model was established by creating the magnetic system model, dividing the mesh, setting the boundary conditions, and applying the load by using the finite element method. The effect of the magnetic field on the particle separation process was analyzed according to the transient equation of motion. Results demonstrate that the magnetic field strength in the axial direction is in good agreement with the theoretical value. The accuracy of calculation model is verified. The magnetic field gradient is maximal near the upper edge of excitation coil in the magnetic separation column. The magnetic field gradient is $466.8 \mathrm{kA} / \mathrm{m}$ per meter when the excitation current is $4 \mathrm{~A}$. The particles form fluxes and accelerate downward. The fluxes begin to disperse and decelerate downward due to the sudden direction change of the magnetic field gradient below the center plane of the coil. The gangue and intergrowth are separated out from the fluxes under the action of water flow. High-grade concentrate is obtained. The proposed model provides evidence for the optimization of the magnetic system in the magnetic separation column.
\end{abstract}

Keywords: Magnetic field, Magnetic separation column, Separation process, Thin solenoid coil

\section{Introduction}

Iron minerals are the most abundant metal mineral resources in the world [1-4]. China's iron ore reserve is extremely large and magnetite ore accounts for more than two-thirds of the total. Magnetic separation is generally adopted in the mineral processing of magnetite ore due to its high specific magnetic susceptibility [5-8]. Magnetic particles passing through the magnetic field regions are magnetized and join together to form fluxes. The gangue and intergrowth are mixed into the fluxes due to magnetic agglomeration. Effectively dispersing the magnetic agglomeration is necessary, as well as removing the gangue and intergrowth mixed in the fluxes, to improve the grade of concentrate. The magnetic separation column is a weak magnetic and gravity concentration equipment that can fully utilize and disperse magnetic agglomeration. At present, magnetic separation columns have been widely used in concentrating mills in China. The grade of concentrate can be increased from $2 \%$ to $7 \%$ and the effect of iron extraction and impurity reduction is evident [9].

However, the magnetite ore tends to be of poor grade, composed of fine particles, and impure, with the continuous development and utilization of mineral resources. The ore must be finely ground and deeply separated. Exploration of the separation process of ore particles is necessary to

*E-mail address: lif970204@163.com

ISSN: $1791-2377$ @ 2019 Eastern Macedonia and Thrace Institute of Technology. All rights reserved. doi:10.25103/jestr.122.08 improve the ability of magnetic separation column to process fine particles, which brings challenges to the study of magnetic separation columns.

Scholars have conducted extensive studies on the magnetic field, operating parameters, and particle separation process in the magnetic separation column [10-17]. However, deviation problems remain in actual working conditions in terms of magnetic field analysis and the effect of magnetic field on the particle separation process. Therefore, accurate calculation of the magnetic field parameters and determining the influence of magnetic field on the force, motion, and separation process of ore particle are urgent problems that need to be solved.

Based on the analysis, this study establishes the calculation model of the magnetic field of the magnetic separation column by using the finite element method (FEM). The magnetic field parameters are calculated according to the electromagnetic field theory. The equation of motion is built to investigate the motion state and separation process of ore particles. Moreover, the influence of magnetic field on the particle separation process is accurately analyzed, which provides a reference for developing and optimizing the magnetic system of the magnetic separation column.

\section{State of the art}

At present, scholars have performed numerous studies on magnetic separation columns and equipments. The process 
flow, as well as the operating and magnetic system parameters, was studied by K. Y. Kim [11] to meet the separation requirements. However, these achievements are only suitable to the specific mineral samples. M. S. Jena [12] and Kyoung-oh Jang [13] explored the influence of magnetic field and operating parameters on the separation results by using experimental methods. However, comprehensive analysis results of magnetic field characteristics were not obtained. The system of magnetic separator was modeled to calculate parameters by using the FEM, such as magnetic field strength and gradient, as well as magnetic force [14-15], but detailed analysis of the particle separation process was unavailable. The magnetic forces of ore particles in the separation space were calculated by using the FEM [16]. Nevertheless, the equation of motion was not established to study the movement of ore particles. Toru Inaba [17] analyzed the magnetic particle trajectories under the action of magnetic field distributed in a permanent magnet, but the method is unsuitable for magnetic fields produced by electromagnetic coils. The motion of magnetic particle in a fluid under the action of an external magnetic field was reduced [18]. However, the magnetic field in the magnetic separation equipment was not analyzed in detail. Guangyao Huang [19] calculated the magnetic field parameters of an excitation coil by using the FEM, but the effect of the magnetic field on the separation process of mineral particles was not derived. A few studies analyze the magnetic field characteristics and particle separation process to improve the shortage of magnetic separation columns. Dianbing Zhu enhanced the performance of the magnetic separation column by introducing the demagnetization process and alternating the magnetic field [20]. The magnetic system was designed according to the experience. The influence of position and spacing of the excitation coils on the separation result was studied using experimental method. However, only the magnetic field strength of four points on the coil axis was measured. Tonglin Zhao [21-22] qualitatively analyzed the separation process of ore particles according to the equation of motion. Nevertheless, only the magnetic field strength along four paths in the magnetic separation column was measured when the excitation current was $12 \mathrm{~A}$. The particle separation process based on the equation of motion was reduced [23], but the quantitative analysis of the particle's magnetic force was unavailable.

Separation experiments using the magnetic separation column have been performed for optimal separation results. Few studies have explored the magnetic field and particle separation process in the magnetic separation column. In this study, the calculation model of the magnetic field of the magnetic separation column was established through FEM. The magnetic field strength and gradient in the axial and radial directions were calculated based on the electromagnetic field theory. Furthermore, the forces of ore particles were calculated. The effect of magnetic field on the particle separation process was reduced by the equation of motion, thereby providing a basis for the optimization of the magnetic system.

The rest of this study is organized as follows. Section 3 establishes the calculation model of the magnetic field and presents the method for calculating the forces of particles. Section 4 discusses the characteristics of the magnetic field and its effect on the particle separation process. Finally, Section 5 summarizes the conclusions.

\section{Methodology}

3.1 Proposed magnetic field calculation model for magnetic separation column

The electromagnetic system of magnetic separation column is composed of groups of thin solenoid coils arranged from top to bottom. The coils are powered in sequence. The structure parameters of coils are set as follows. The inner radius $r_{1}$ is $22 \mathrm{~mm}$, the outer radius $r_{2}$ is $42 \mathrm{~mm}$, the height $L$ is $25 \mathrm{~mm}$, and the number of turns $N$ is 320 . The coils are made from an enameled copper wire with diameter of 1.2 $\mathrm{mm}^{2}$. The relative magnetic permeability is 1 . The calculation model of the electromagnetic field of the magnetic separation column is established via finite element software. Only half of the area of the coil is modeled due to the axisymmetric feature of the coil. PLANE53 element is selected to simulate the air and coil, whereas INFIN110 element is selected to simulate the infinite boundary. The relative magnetic permeability of air and coil is 1 and the resistivity of coil is $3 \times 10^{-8} \Omega \mathrm{m}$. The quadrilateral meshes are generated via map method in the areas of coil and infinite boundary, whereas the triangular meshes are generated via free method in the area of air. The meshes are densified in the local areas. The flag is applied to infinite boundary, the current load is applied to the coil, and 2D static analysis is conducted. The results of magnetic field parameters in the axial and radial directions are extracted and displayed after the solution is obtained. The magnetic field strength determined from the simulation is compared with the theoretical value to verify the correctness of the proposed calculation model.

Based on the electromagnetic field theory, the magnetic field strength on the axis of the multi-layer solenoid coil operating under direct current is:

$$
\begin{gathered}
H_{z}=\frac{1}{2} \frac{N I}{\left(r_{2}-r_{1}\right) L}\left[\left(\frac{L}{2}+z\right) \ln \frac{r_{2}+\sqrt{r_{2}^{2}+\left(\frac{L}{2}+z\right)^{2}}}{r_{1}+\sqrt{r_{1}^{2}+\left(\frac{L}{2}+z\right)^{2}}}\right. \\
\left.+\left(\frac{L}{2}-z\right) \ln \frac{r_{2}+\sqrt{r_{2}^{2}+\left(\frac{L}{2}-z\right)^{2}}}{r_{1}+\sqrt{r_{1}^{2}+\left(\frac{L}{2}-z\right)^{2}}}\right]
\end{gathered}
$$

where $H_{z}$ is magnetic field strength at the point $z$ on the axis, $A / m ; r_{1}$ is inner radius of the coil, $m ; r_{2}$ is outer radius of the coil, $m ; L$ is height of the coil, $m$; $I$ is current intensity of the coil, $A$; and $N$ is number of the coil turns.

\subsection{Equation of motion for ore particle}

The magnetic particles or fluxes in the separation space are subjected to effective gravity and flow resistance in addition to magnetic force. Only axial force and motion of particle or flux are analyzed to simplify the calculation.

The effective gravity of ore particle is:

$$
F_{g}=\frac{\pi}{6} d^{3}(\delta-\rho) g
$$

where $F_{g}$ is effective gravity of the ore particle, $N ; \delta$ is density of the ore particle, $\mathrm{kg} / \mathrm{m}^{3} ; \rho$ is density of the 
water, $\mathrm{kg} / \mathrm{m}^{3} ; d$ is diameter of the ore particle, $\mathrm{m}$; and $g$ is gravity acceleration, $\mathrm{m} / \mathrm{s}^{2}$.

The magnetic force of the ore particle can be expressed as:

$$
F_{c}=\frac{\pi}{6} d^{3} \mu_{0} \chi \delta H g r a d H
$$

where $F_{c}$ is the magnetic force of the ore particle, $N ; \mu_{0}$ is the magnetic permeability of vacuum, $N / A^{2} ; \chi$ is the specific magnetic susceptibility of the ore particle, $\mathrm{m}^{3} / \mathrm{kg}$; $H$ is magnetic field strength, $A / m$; and $\operatorname{grad} H$ is magnetic field gradient, $A / \mathrm{m}^{2}$.

The flow resistance is:

$$
F_{r}=k\left(\frac{\pi}{6} d^{3}\right)^{2 / 3}\left(v+\frac{v_{a}}{1-\lambda}\right)^{2} \rho
$$

where $F_{r}$ is flow resistance, $N ; k$ is coefficient related to the ore shape and water flow pattern; $v$ is velocity of the ore particle along the axis, $m / s ; v_{a}$ is velocity of the water flow, $\mathrm{m} / \mathrm{s} ;$ and $\lambda$ is bulk density.

The magnetic forces of ore particles are calculated according to the magnetic field strength and gradient obtained in Section 3.1.

The magnetic particles in the separation space accelerate downward under the action of effective gravities and magnetic forces and the velocity $v$ increases. A dynamic system is formed and the bulk density $\lambda$ gradually increases from top to bottom. As the space is occupied by the ore particles, the relative cross-sectional area becomes small, whereas the total water flow remains constant. The velocity of water flow $v_{a}$ becomes fast. Calculating the flow resistance is difficult due to the variation of water velocity. The relative motions of magnetic and non-magnetic particles are qualitatively analyzed.

The equation of motion for the ore particle is:

$$
\begin{aligned}
& m \frac{d v}{d t}=F_{g}+F_{c}-F_{r} \\
= & \frac{\pi}{6} d^{3}(\delta-\rho) g+\frac{\pi}{6} d^{3} \mu_{0} \chi \delta H g r a d H \\
& -k\left(\frac{\pi}{6} d^{3}\right)^{2 / 3}\left(v+\frac{v_{a}}{1-\lambda}\right)^{2} \rho
\end{aligned}
$$

When the force balances, the formula $m \frac{d v}{d t}=0$ holds [23]. The velocity of the ore particle can be simplified as:

$$
v=A_{0}\left\{\left[A_{1} d(\delta-\rho)+A_{2} d \chi \delta H g r a d H\right]^{1 / 2}-v_{a}\right\}
$$

where

$$
A_{0}=\frac{1}{1-\lambda}
$$

$$
A_{1}=(1-\lambda)^{2} \frac{1}{k \rho}\left(\frac{\pi}{6}\right)^{1 / 3} g
$$

$$
A_{2}=(1-\lambda)^{2} \frac{1}{k \rho}\left(\frac{\pi}{6}\right)^{1 / 3} \mu_{0}
$$

The density of magnetite ore $\delta_{1}$ is larger than those of intergrowth $\delta_{2}$ and gangue $\delta_{3}$. The specific magnetic susceptibility of magnetite ore $\lambda_{1}$ is also larger than those of intergrowth $\lambda_{2}$ and gangue $\lambda_{3}$. Thus, the rate of water flow can be controlled to meet the following conditions:

$$
v_{a}>\left[A_{1} d\left(\delta_{2}-\rho\right)+A_{2} d \chi_{2} \delta_{2} H g r a d H\right]^{1 / 2}
$$

$v_{a}<\left[A_{1} d\left(\delta_{1}-\rho\right)+A_{2} d \chi_{1} \delta_{1} H g r a d H\right]^{1 / 2}$

The magnetic particles sink, whereas the gangue and intergrowth with the same size float and become tailings under the action of water flow. The grade of concentrate is improved.

\section{Result Analysis and Discussion}

\subsection{Magnetic field analysis of magnetic separation column}

The magnetic field parameters of the magnetic separation column are calculated according to the calculation model proposed in Section 3.1. The magnetic field strength obtained by the finite element software and from Formula (1) is shown in Fig. 1. The excitation current is $2 \mathrm{~A}$. The axis distance ranges from $-0.025 \mathrm{~m}$ to $0.025 \mathrm{~m}$, that is, from $0.025 \mathrm{~m}$ below the center point of coil to $0.025 \mathrm{~m}$ above the center point. Evidently, the simulation results are in good agreement with the theoretical values. The results indicate that the calculation model can reflect the real magnetic field characteristics of the coil. Accuracy of the model is verified.

The cloud picture of the magnetic field strength is shown in Fig. 2. Near the center of the coil, the magnetic field strength is large, whereas the magnetic field gradient is small. The magnetic field is close to uniform. The magnetic field strength gradually decreases, whereas the magnetic field gradient increases from the center to the upper and lower edges of the coil along the axial direction. Meanwhile, the magnetic field strength gradually increases and the magnetic field gradient also increases from the center to the inner wall of the coil along the radial direction.

The horizontal plane where the center of the coil is located is defined as the reference plane to conveniently evaluate the magnetic field strength and gradient in the axial and radial directions of the coil. The parallel horizontal

planes ${ }^{z_{0}}(\mathrm{z}=0), z_{1}(z=1 / 4 L), z_{2}(z=1 / 2 L)$, and $z_{3}(z=$ $3 / 4 \mathrm{~L})$ are set with a spacing of $1 / 4 L(6.25 \mathrm{~mm})$ along the axial direction. Vertical planes intersecting with the horizontal planes $x_{0}(x=0), x_{1}\left(x=1 / 4 r_{1}\right), x_{2}\left(x=1 / 2 r_{1}\right)$ and $x_{3}\left(x=3 / 4 r_{1}\right)$ are set with a spacing of $1 / 4 r_{1}(5.5 \mathrm{~mm})$ in the radial direction. The excitation current of coil is set as $1,2,3,4$, and $5 \mathrm{~A}$. The magnetic field strength in the four horizontal planes $z_{0}, z_{1}, z_{2}$, and $z_{3}$ is shown in Fig. 3 . The magnetic field strength increases as the distance from 
the center increases. The value is $9.5 \mathrm{kA} / \mathrm{m}$ at the center of coil and $13 \mathrm{kA} / \mathrm{m}$ near the inner wall of coil in the $z_{0}$ plane. The magnetic field gradient increases as the radial distance increases. The average value is $159 \mathrm{kA} / \mathrm{m}^{2}$ in the $z_{0}$ plane, whereas the values are $156 \mathrm{kA} / \mathrm{m}^{2}$ and $106 \mathrm{kA} / \mathrm{m}^{2}$ in the $z_{1}$ and $z_{2}$ planes, respectively. The farther the distance from the reference plane, the smaller the magnetic field gradient becomes. The magnetic field strength in the four vertical planes $x_{0}, x_{1}, x_{2}$, and $x_{3}$ is shown in Fig. 4 . The magnetic field strength is symmetrical to the reference plane, which is maximal at the center of the path and decreases as the axial distance increases. The magnetic field strength is positive, that is, its direction is from bottom to top. Near the upper edge of the coil, the magnetic field gradient is large and the average value is $233 \mathrm{kA} / \mathrm{m}^{2}$ (in the $x_{0}$ plane). Near the center of the coil, the magnetic field gradient decreases to $28 \mathrm{kA} / \mathrm{m}^{2}$ (in the $x_{0}$ plane). Fig. 3 and Fig. 4 show that the magnetic field gradient in the axial direction plays a key role in the vicinity of the upper edge of the coil. The magnetic particle is mainly subjected to the axial magnetic force. The radial magnetic field gradient increases near the center of the coil, where the magnetic particle is mainly subjected to the radial magnetic force. The magnetic field gradient in the lower side of the reference plane is similar to that in the upper side but in the opposite direction.

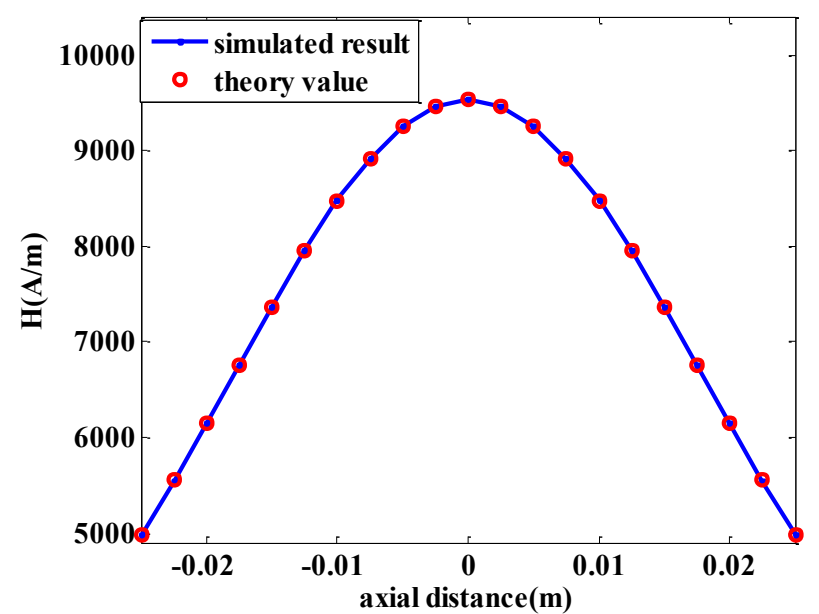

Fig. 1. Magnetic field strength on the axis of coil

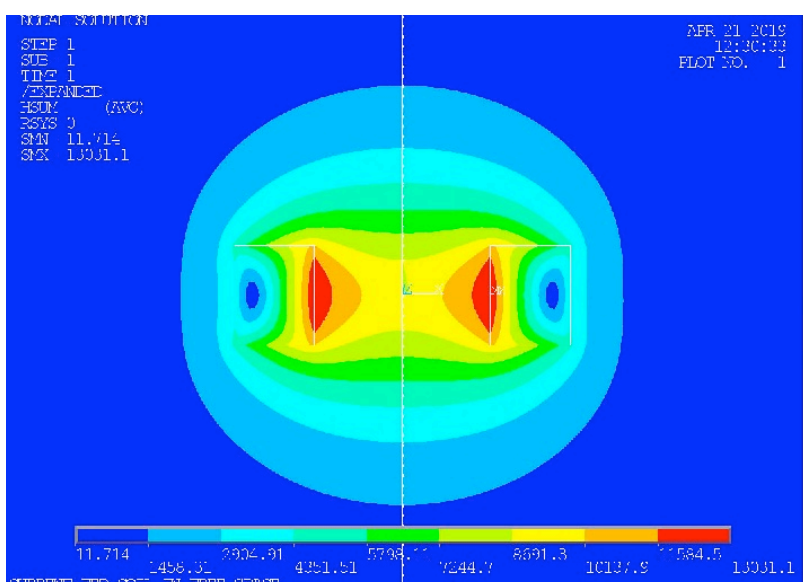

Fig. 2. Cloud picture of magnetic field strength

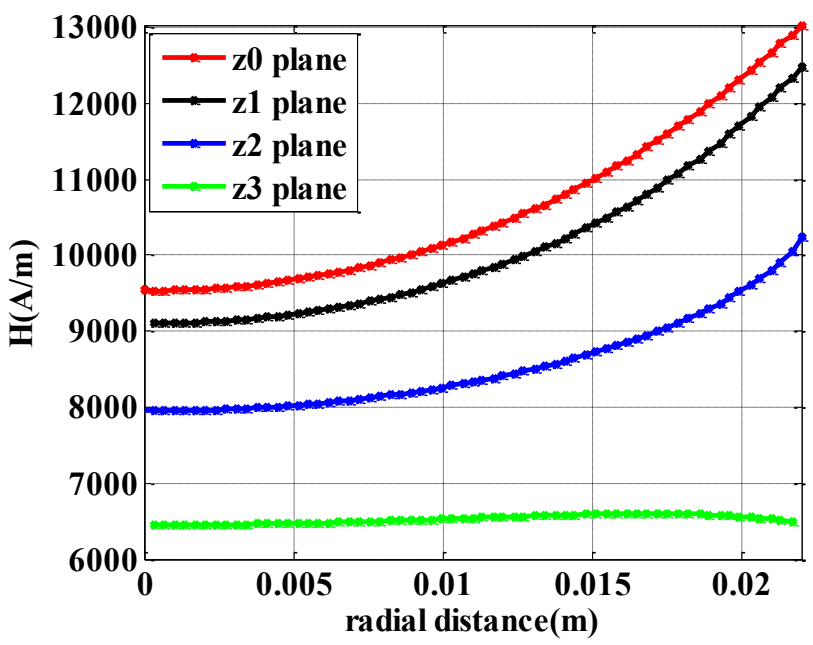

Fig. 3. Magnetic field strength in horizontal planes

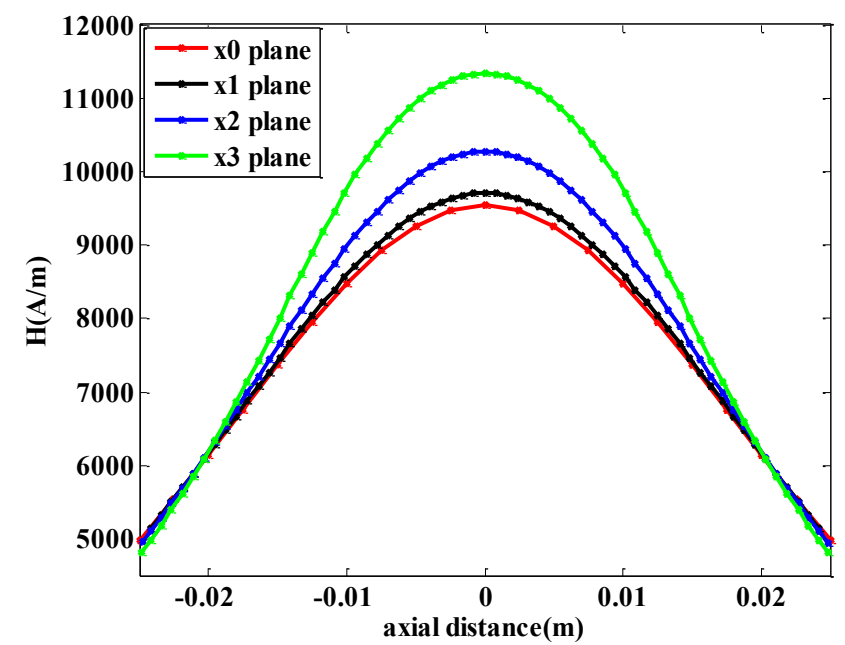

Fig. 4. Magnetic field strength in vertical planes

4.2 Effect of magnetic field on particle separation process The forces of ore particle are analyzed according to the method described in Section 3.2. The magnetic force is determined by the magnetic field strength and gradient as shown in Equation (3). Therefore, the product, $H \operatorname{grad} H$, can be used to quantitate the magnitude of the magnetic force. The curves of $H \operatorname{grad} H$ are shown in Fig. 5 when the DC current in the coil is set as $1,2,3,4$, and $5 \mathrm{~A}$.

The magnetic forces are positive below the reference plane and negative on the other side, that is, the magnetic forces point to the direction of the reference plane. The magnetic force is zero at the center of the coil and is maximal at the places of $-0.125 \mathrm{~m}$ and $0.125 \mathrm{~m}$, where the upper and lower edges of the coil are located. When the excitation current is $4 \mathrm{~A}$, the magnetic field strength is 14.7 $\mathrm{kA} / \mathrm{m}$, the magnetic field gradient is $466.8 \mathrm{kA} / \mathrm{m}^{2}$, and the product $H \operatorname{grad} H$ is $6.88 \times 10^{9} \mathrm{~A}^{2} / \mathrm{m}^{3}$ near the upper edge of the coil. The magnetic forces increase with the increase of the current. The magnetic forces and effective gravities of different-sized particles near the upper edge of the coil are calculated when the excitation current is $2,3,4$, and $5 \mathrm{~A}$. The density of magnetite ore $\rho_{1}$ is $5.17 \times 10^{3} \mathrm{~kg} / \mathrm{m}^{3}$ [18], and the specific magnetic susceptibility $\chi$ is $1.21 \times 10^{-3}$ $\mathrm{m}^{3} / \mathrm{kg}$. The results are shown in Fig. 6. Evidently, the magnetic forces are higher than the effective gravities when the excitation current is above $3 \mathrm{~A}$. The smaller the particle 
size is, the smaller is the difference between the magnetic force and effective gravity.

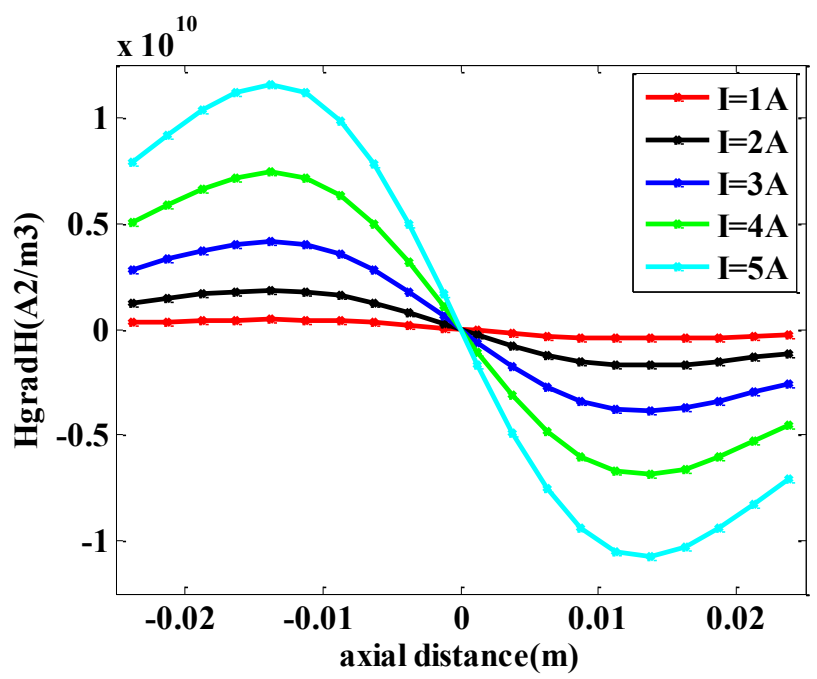

Fig. 5. $H \operatorname{grad} H$ under different currents

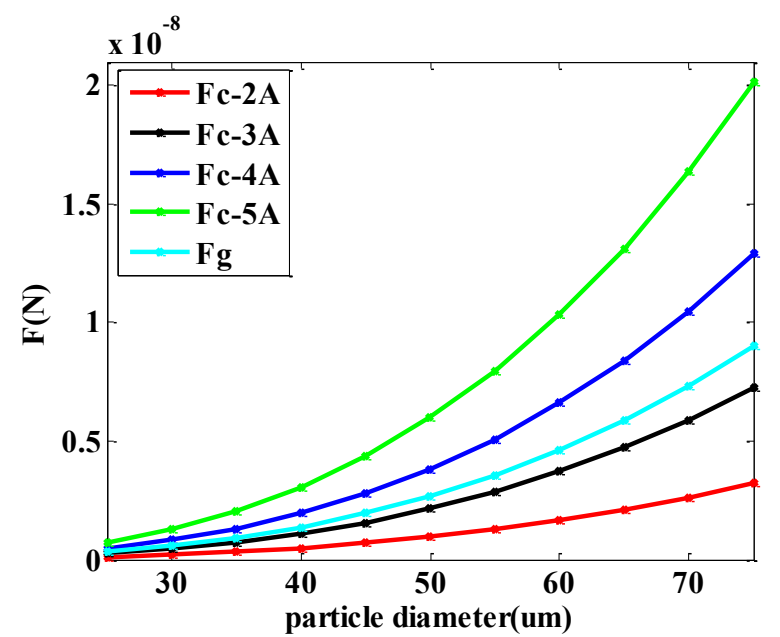

Fig. 6. Magnetic forces and effective gravities of different-sized particles

The ore particles are not affected by the magnetic field and settle down under the action of effective gravity in the space above the excitation coil. The magnetic particles agglomerate and rapidly form fluxes along the magnetic field lines near the upper edge of the coil. The fluxes overcome the flow resistance and accelerate downward. The settling velocity reaches the maximum near the center of the coil. The fluxes begin to be subjected to the upward magnetic forces below the center plane. The particles disperse due to the sudden direction change of magnetic forces. The intergrowth and gangue are separated from the fluxes and finally become tailings under the action of water flow. The fluxes continue to decelerate downward. The magnetic field in the magnetic separation column causes the particles to agglomerate, disperse, and agglomerate again. The fluxes are purified and eventually become high-grade concentrate.

\section{Conclusion}

A calculation model of the magnetic field of the magnetic separation column was developed based on the FEM to explore the effect of magnetic field on the particle separation process. Magnetic field strength and gradient, as well as magnetic force, were calculated by numerical simulation technology. The effect of the magnetic field on the particle separation process was analyzed. The following conclusions could be drawn:

(1) The magnetic field strength is large, whereas the magnetic field gradient is small near the center of the coil. The magnetic field is close to uniform. The magnetic field strength decreases, the magnetic field gradient increases, and the product $H \operatorname{grad} H$ increases from the center to the upper and lower edges of the coil along the axial direction. The magnetic force points toward the direction of the coil's center plane. The magnetic field strength and gradient gradually increases from the center to the inner wall of the coil along the radial direction.

(2) The magnetic force near the upper edge of the coil is large and the ore particles agglomerate to form fluxes and accelerate downward. The fluxes begin to be subjected to the upward magnetic forces and disperse below the center plane of the coil. The gangue and intergrowth are separated out from the fluxes under the action of water flow. The magnetic field generated by groups of coils in the magnetic separation column causes the fluxes to agglomerate, disperse, and agglomerate again. The fluxes are purified and eventually become high-grade concentrate.

Thus, the magnetic force, effective gravity, and flow resistance are considered in the proposed method to accurately evaluate the particle separation process. However, the separation process is influenced by many forces. Other influencing factors, such as magnetic agglomeration, should be considered in future studies.

\section{Acknowledgements}

This work was supported by Natural Science Foundation of Liaoning Province of China(Grant No. 20170540720)

This is an Open Access article distributed under the terms of the Creative Commons Attribution License

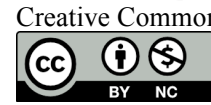

\section{References}

1. A. Simakov, V. E. Isaev, "X-Ray fluorescence determination of magnetite iron in iron ores after preliminary magnetic separation". Inorganic Materials, 51(14), 2015, pp.1438-1441.

2. Jianwen Yu, Yuexin Han, Yanjun Li, Peng Gao, "Effect of magnetic pulse pretreatment on grindability of a magnetite ore and its implication on magnetic separation". Journal of Central South University, 23(12), 2016, pp.3108-3114.
3. Hao Hu, Jianwei Li, David Lentz, Zhe Ren, Xinfu Zhao, Xiaodong Deng, Douglas Hall, "Dissolution-reprecipitation process of magnetite from the Chengchao iron deposit: insights into ore genesis and implication for in-situ chemical analysis of magnetite". Ore Geology Reviews, 57, 2014, pp.393-405.

4. Saikat Samanta, Siddhartha Mukherjee, Rajib Dey, "Oxidation behaviour and phase characterization of titaniferous magnetite ore of eastern India". Transactopms of Nonferrous Metals Society of China, 24(9), 2014, pp.2976-2985. 
5. Jianwen Yu, Yuexin Han, Yanjun Li, "Beneficiation of an iron ore fines by magnetization roasting and magnetic separation". International Journal of Mineral Processing, 168, 2017, pp.102108.

6. Sergey N. Podoynitsyn, Olga N. Sorokina, Alexander L. Kovarski, "High-gradient magnetic separation using ferromagnetic membrane". Journal of Magnetism and Magnetic Materials, 397, 2016, pp.51-56.

7. M. Iranmanesh, J. Hulliger, "Magnetic separation: its application in mining, waste purification, medicine, biochemistry and chemistry". Chemical Society Reviews, 46(19), 2017, pp.5925-5934.

8. Naiyang Ma, Joseph Blake Houser, "Recycling of steelmaking slag fines by weak magnetic separation coupled with selective particle size screening". Journal of Cleaner Production, 82, 2014, pp.221231.

9. Zhian Liang, Qing Xia, Hongqiang Wu, "Development and application of several magnetic equipments". Metal Mine, (2), 2017, pp.128-134.

10. Haihuan Zhou, "Test of improving the quality and reducing impurity for a ultralow-grade magnetite in Hebei”. Nonferrous Metals(Mineral Processing Section), (1), 2016, pp.52-55.

11. K. Y. Kim, E. J. Jang, D. Y. Kim, S. M. Jung, "Effect of magnetic field on reduction of magnetite". Ironmaking \& Steelmaking, 44(1), 2017, pp.6-16.

12. M. S. Jena, H. K. Tripathy, J. K. Mohanty, J. N. Mohanty, S. K. Das, P. S. R. Reddy, "Roasting followed by magnetic separation: a process for beneficiation of titano-magnetite ore". Separation Science and Technology, 50(8), 2015, pp.1221-1229.

13. Kyoung-oh Jang, Venkata R. M. Nunna, Sarath Hapugoda, Anh V. Nguyen, Warren J. Bruckard, "Chemical and mineral transformation of a low grade goethite ore by dehydroxylation, reduction roasting and magnetic separation". Minerals Engineering, 60, 2014, pp.14-22.
14. S. K. Baik, D. W. Ha, J. M. Kwon, Y. J. Lee, R. K. Ko, "Magnetic force on a magnetic particle within a high gradient magnetic separator". Physica C: Superconductivity, 484, 2013, pp.333-337.

15. S. K. Baik, D. W. Ha, R. K. Ko, "Magnetic field analysis of high gradient magnetic separator via finite element analysis". Physica C: Superconductivity, 480, 2012, pp.111-117.

16. Panpan Fan, Minqiang Fan, An Liu, "Using an axial electromagnetic field to improve the separation density of a dense medium cyclone". Minerals Engineering, 72, 2015, pp.87-93.

17. Toru Inaba, Taku Sakazume, Yoshihiro Yamashita, Shinya Matsuoka, "Magnetic particle movement program to calculate particle paths in flow and magnetic fields". Journal of Magnetism and Magnetic Materials, 351, 2014, pp.37-46.

18. J. Ravnik, M. Hriberšek, "High gradient magnetic particle separation in viscous flows by 3D BEM". Computational Mechanics, 51(4), 2013, pp.465-474.

19. Guangyao Huang, Xiangyu Gu, Yuchuan Cao, "Properties of magnetic field in separation chamber for magnetic flotation generated by electric coils". Mining and Metallurgical Engineering, 36(3), 2016, pp.31-35.

20. Dianbing Zhu, Jujian Zhu, Tonglin Zhao, "Study on the structure improvement and optimization of magnetic separation column". China Mining Magazine, 25(5), 2016, pp.121-123, 128.

21. Tonglin Zhao, Zhonghang Chen, Guangzhen Chen, "Characteristic analysis and application of the separation of magnetic separation column". Multipurpose Utilization of Mineral Resources, (3), 2013, pp.15-17.

22. Zhonghang Chen, Tonglin Zhao, Guangzhen Chen, "Application and practice of the combined thick coils in magnetic separators". Multipurpose Utilization of Mineral Resources, (5), 2012, pp.50-53.

23. Guangzhen Chen, Bingyu Liu, Wei Zhou, Longxi Zheng, "Magnetic separation column and its commercial application". Metal Mine, (9), 2002, pp.30-31, 43. 